\title{
Spatial and temporal episodic memory retrieval recruit dissociable functional networks in the human brain
}

\author{
Arne D. Ekstrom and Susan Y. Bookheimer ${ }^{1}$ \\ Center for Cognitive Neurosciences, Semel Institute, Department of Psychiatry and Biobehavioral Sciences, \\ UCLA School of Medicine, Los Angeles 90095, California, USA
}

\begin{abstract}
Imaging, electrophysiological studies, and lesion work have shown that the medial temporal lobe (MTL) is important for episodic memory; however, it is unclear whether different MTL regions support the spatial, temporal, and item elements of episodic memory. In this study we used fMRI to examine retrieval performance emphasizing different aspects of episodic memory in the context of a spatial navigation paradigm. Subjects played a taxi-driver game ("yellowcab"), in which they freely searched for passengers and delivered them to specific landmark stores. Subjects then underwent fMRI scanning as they retrieved landmarks, spatial, and temporal associations from their navigational experience in three separate runs. Consistent with previous findings on item memory, perirhinal cortex activated most strongly during landmark retrieval compared with spatial or temporal source information retrieval. Both hippocampus and parahippocampal cortex activated significantly during retrieval of landmarks, spatial associations, and temporal order. We found, however, a significant dissociation between hippocampal and parahippocampal cortex activations, with spatial retrieval leading to greater parahippocampal activation compared with hippocampus and temporal order retrieval leading to greater hippocampal activation compared with parahippocampal cortex. Our results, coupled with previous findings, demonstrate that the hippocampus and parahippocampal cortex are preferentially recruited during temporal order and spatial association retrieval-key components of episodic "source" memory.
\end{abstract}

Although there is little doubt in cognitive neuroscience regarding the importance of the human medial temporal lobes-especially the hippocampal area-in declarative memory processes (Scoville and Milner 1957; Zola-Morgan et al. 1994; Vargha-Khadem et al. 1997), the exact role of the hippocampus and parahippocampal gyrus in memory retrieval remain under intense debate (Squire et al. 2004). Aggleton and Brown (2006) argue for a distinction between hippocampus and perirhinal cortex (part of anterior parahippocampal gyrus) in processing based on item familiarity and recovery of the source from which the item was learnedprocesses that may be carried out by perirhinal cortex and hippocampus, respectively. Put another way, the hippocampus may primarily code episodic or source-rich information, while perirhinal cortex codes familiarity-based information about stimuli. Davachi et al. (2003), however, outlined a role for the hippocampus and posterior parahipppocampal gyrus (parahippocampal cortex) in source retrieval using fMRI-specifically, the conditions under which an item was originally encoded-compared with perirhinal cortex, which was most active during item retrieval only. Source information may encompass both spatial and temporal components, e.g., remembering when in time an item was learned, or remembering where in space the item was learned (Tulving 1993; Nyberg et al. 1996; Fujii et al. 2004). Thus, it is possible that the role of the parahippocampal cortex and hippocampus may depend on the type of source information being retrieved. Little is known about the role of the hippocampus and parahippocampal cortex in recovering different types of source information. The present study sought to address this important issue.

\footnotetext{
${ }^{1}$ Corresponding author.

E-mail sbook@ucla.edu; fax (310) 794-7406.

Article is online at http://www.learnmem.org/cgi/doi/10.1101//m.575107.
}

Several fMRI studies suggest the importance of the parahippocampal cortex in spatial processing. Epstein and Kanwisher (1998) found increased activation of the parahippocampal cortex while subjects viewed scenes compared with viewing faces or other objects. Arguing for the importance of the parahippocampal cortex in spatial navigation, Aguirre et al. (1996) showed increased parahippocampal activation during navigation compared with a rote control task where subjects navigated back and forth down a corridor. The fact that subjects likely relied heavily on landmarks during the active navigation condition suggested that the parahippocampal activations observed by Aguirre and colleagues could relate to integrating landmark information with navigational routes. Indeed, lesions of parahippocampal cortex impair recall for recently learned spatial routes (Bohbot et al. 1998). Also arguing for the involvement of the parahippocampal cortex in landmark representation during navigation, Janzen and van Turenout (2004) had subjects navigate a virtual environment and then perform a recognition task to determine what aspects of navigation most activated the parahippocampal cortex. Subjects showed the greatest activation when viewing previously seen objects that were at navigational decision points, and therefore served as spatial landmarks, compared with objects that received equal amounts of attention but were not at decision points.

Other studies argue for the importance of the hippocampus and retrosplenial cortex in spatial representation. Lesions of the right hippocampus, in particular, impair patients' memory for the locations of objects in arena independent of object identity (Stepankova et al. 2004). Following navigation of a virtual environment, patients with left hippocampal damage are deficient in recalling contextual aspects of events such as whom they met during navigation, places visited, and the order of events (Spiers et al. 2001). These data together argue for parahippocampal in- 
volvement in landmark representation and hippocampal involvement in episodic memory representation such as spatial and temporal context (Burgess et al. 2002). Other studies, though, argue for the importance of retrosplenial cortex in spatial representation compared with the hippocampus (Maguire 2001; Shelton and Gabrieli 2002; Rosenbaum et al. 2004). Wolbers and Buchel (2005) found that the hippocampus activated most strongly during acquisition of spatial information in the first few trials of navigation, while the retrosplenial cortex correlated with long-term learning and recall of spatial layouts over navigation sessions (Wolbers and Buchel 2005). The roles of the parahippocampal cortex, retrosplenial cortex, and hippocampus in recall of spatial versus temporal episodic components of memory retrieval from a recent navigational experience, however, have yet to be tested.

To address the issue of hippocampal, perirhinal, parahippocampal, and extra-hippocampal activations in episodic memory retrieval, we controlled the degree to which subjects acquired spatial and temporal information during navigation of a virtual environment. We did this by having subjects play a taxi-driver like virtual navigation game, in which they freely searched for passengers and delivered them to specific stores, tasks our previous results have shown to strongly activate the hippocampus and parahippocampal cortex at both the single neuron and ensemble level (Ekstrom et al. 2003, 2005). Subjects met specific passengers with unique identities and then delivered them to specific stores. We then tested subjects' memories for objects experienced during navigation and for associations formed during navigation, with our principle focus on how specific retrieval processes would differentially recruit brain regions during remembering of a recently experienced spatial environment.

Subjects performed three retrieval tasks following navigation while undergoing fMRI imaging. Subjects first viewed familiar and novel landmarks and made judgments about whether they had seen these objects before or not. This task involved simple familiarity-based judgments, serving as a contrast for subsequent tasks that involved associative judgments. Subjects then performed spatial and temporal association retrieval, in which they made judgments about whether they had delivered a passenger to a store and the order that passengers were delivered. Based on previous results and the implication of both the hippocampus and parahippocampal cortex in spatial processing (Burgess et al. 2002; King et al. 2005), we predicted the strongest activation in both regions during retrieval of spatial associations. We expected temporal order judgments to activate the hippocampus to a greater extent than the parahippocampal gyrus, based on the hypothesized role of the hippocampus but not the parahippocampal gyrus in episodic memory (Tulving 1993). Finally, we predicted that item recognition would show the least hippocampal activation but significant parahippocampal and perirhinal cortex activation, because previous studies suggest the involvement of the parahippocampal gyrus but not hippocampal involvement in familiarity-based recognition processes (Yonelinas et al. 2002; Holdstock et al. 2005). ditions across subjects.

\section{Materials and Methods}

\section{Experimental design}

A total of 14 paid right-handed volunteer subjects (seven male, seven female), fluent in English, were recruited with no history of neurological illness and participated as subjects. All recruitment procedures conformed to UCLA IRB testing protocols. The experimental task involved two separate components. Immediately prior to scanning, subjects navigated a virtual reality (VR) environment in a taxi-driver like game ("yellowcab"), in which they encountered passengers and delivered them to stores. Subjects then performed three separate recognition tasks within the scanner. The virtual city was composed of a $5 \times 5$ grid containing roads, stores to which the subject delivered passengers, and buildings the subject did not visit (Fig. 1A,B). The city contained four different stores and 21 buildings. Subjects navigated the city using a joystick; the virtual town was presented using pyepl and yellowcab2 (download http://memory.psych.upenn.edu/ programming/experiments/yellowcab2.tgz). If subjects could not find a passenger after $30 \mathrm{sec}$, a brightly colored beam appeared above the passenger to illuminate its location. This ensured that subjects encoded an equal number of passengers and locations. Subjects delivered a total of 12 passengers; thus, each store was delivered to three different times. The navigation part of the experiment was designed to take subjects $\sim 20 \mathrm{~min}$.

Subjects then entered the scanner and began the imaging part of the experiment $\sim 10$ min after completing navigation. (Although all efforts were made to control the time at which subjects began the scan, occasionally subjects required a minute or two of extra preparation.) Subjects responded using an MRIcompatible button pad in their right hand. The experiment was set up as event related, with three runs comprising the three retrieval conditions. In the first run, the landmark recognition condition (Fig. 1C), subjects viewed four familiar and four novel stores randomly shuffled together and repeated three times each for a total of 24 presentations. Our previous findings from human single-neuron recordings during yellowcab2 in hippocampus
A
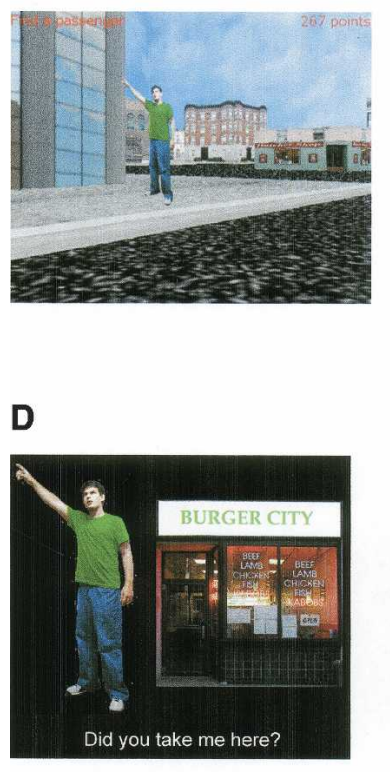

B

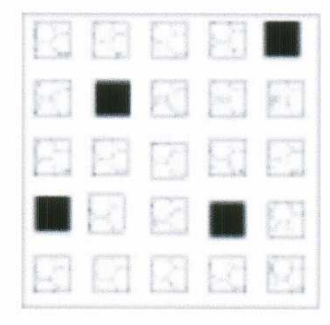

E

C

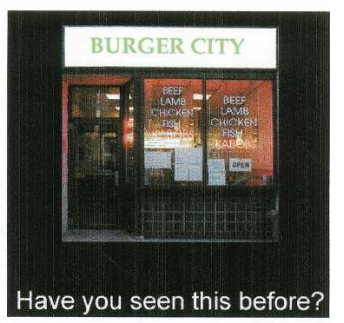

$\mathbf{F}$

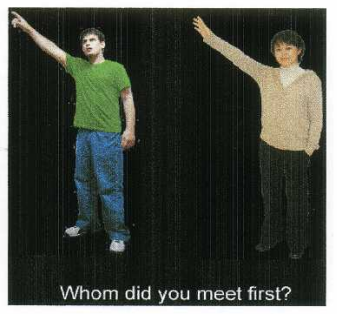

Figure 1. Behavioral methods and results. $(A)$ Screen shot of a passenger waiting on sidewalk from a virtual city that subjects explored. $(B)$ Overview layout of location of stores in virtual city; stores are labeled in black, buildings labeled in white. ( $C-E$ ) Following navigation, subjects performed an episodic retrieval task in the scanner composed of three different conditions: $(C)$ a landmark recognition condition, $(D)$ a spatial association retrieval condition, and $(E)$ a temporal order retrieval condition (see Materials and Methods). ( $F$ ) Mean performance was above chance (50\%; red-dotted line) in all con- 
and parahippocampal cortex suggested that repeating presentations of landmarks would not affect the neural firing rate to a significant extent (Ekstrom et al. 2003). Events in the landmark recognition condition therefore consisted of whether the stimulus was seen during navigation or not; subjects indicated with a button press whether they had seen the stimulus before or not. Events in the landmark recognition condition preceded associative recognition conditions and thus could not be counterbalanced with the associative recognition conditions. This is because the associative recognition conditions used familiar stimuli only, thus providing information about the landmark recognition condition. We address the possible consequences of order effects in the Discussion section.

During the next run, subjects participated in either the spatial or temporal association condition, depending on the counterbalancing condition of the subject (half of the subjects received the spatial association condition first, the other half received the temporal order condition first). During the spatial association condition (Fig. 1D), subjects viewed pairings of passengers with stores; half of the pairings were targets (correct passenger-store pairings), while the other half were lures (incorrect passenger-store pairings). Events thus consisted of whether the passenger-store pairing was novel or familiar. As during the landmark recognition run, subjects indicated with a keypress whether they had experienced the passenger-store pairing previously or not. Subjects viewed a total of 12 familiar store-passenger pairings and 12 rearranged store-passenger pairings based on the original 12 passenger deliveries in the navigation part of the experiment.

During the temporal order condition (Fig. 1E), subjects viewed pairings of passengers adjacent to each other. The events consisted of whether the ordering of passengers was consistent with what was seen previously or whether the passengers were rearranged. Subjects received equal numbers of novel and familiar orderings. Passenger pairings were derived such that the distance in time between passengers was overall balanced to the extent allowed by the experimental design. In other words, subjects saw equal numbers of distantly spaced passengers in time compared with those closely spaced in time. Subjects indicated with a keypress as to which passenger came first in the order of deliveries, the one on the right or the one on the left. Subjects saw 12 passenger pairs arranged such that the more recently experienced passenger occurred on the left and 12 passenger pairs arranged such that the more recently experienced passenger came on the right.

In all retrieval conditions, each target stimulus appeared on the screen for $3 \mathrm{sec}$. Following each stimulus, subjects performed a control task in which they saw even and odd numbers for $6 \mathrm{sec}$ and indicated with a button press whether the number that appeared was odd or even. This control task allowed us to directly contrast recognition with a task that did not spontaneously engage mnemonic processes and thus provided minimal hippocampal activation (Stark and Squire 2001). The timing and control task were the same in all three conditions.

Although the timing of stimuli was identical in all three conditions, the number of stimuli that appeared on the screen in the landmark recognition condition was fewer than in the associative recognition conditions (one vs. two stimuli, see Fig. 1, C vs. D and E). This was necessary because we wished to contrast simple familiarity-based recognition with associative recognition, requiring recovery of previously learned spatial and temporal information (e.g., see Holdstock et al. 2005). An additional stimulus on the screen during landmark recognition would have provided a significant distraction to the subject and likely recruited different brain regions from those involved in familiaritybased recognition. To attempt to deal with this issue to the greatest extent possible within the constraints of our experimental questions, subjects were explicitly instructed to focus on judgments regarding the stimuli rather than features of the stimuli themselves. To facilitate this process, text appeared below each stimulus set in each condition explicitly instructing the subject what their task was (e.g., "Have you seen this before?" "Did you take me here?" "Whom did you meet first?"). We discuss further in the Discussion section the possible impact that the differential number of stimuli may have had on our findings.

Instructions prior to retrieval explicitly instructed subjects to answer questions based on their experience during VR navigation prior to the scan and not based on whether the stimuli had been seen before during retrieval. Because passengers were seen once during navigation compared with stores, which were seen repeatedly during navigation, we did not include them during the recognition part of the experiment.

\section{Imaging procedures}

Using a Siemens Allegra 3T scanner, we collected whole-brain fMRI scans using a gradient-echo, echo-planar scan sequence while the participants performed the retrieval conditions (3- $\mathrm{mm}$ slices; $64 \times 64(3.1 \times 3.1 \mathrm{~mm})$ in-plane resolution; field of view $($ FOV $)=200 \times 200$; repetition time $(\mathrm{TR})=3000 \mathrm{msec}$; echo time $(\mathrm{TE})=35 \mathrm{msec}$; flip angle $(\mathrm{FA})=90)$. Scans were acquired perpendicular to the long axis of the hippocampus to maximize signal intensity in the medial temporal lobes. We acquired highresolution spin-echo scans $(128 \times 128$, e.g., $1.6 \times 1.6 \mathrm{~mm}$, inplane resolution; $\mathrm{FOV}=200 \times 200 ; \mathrm{TR}=5000 \mathrm{msec} ; \mathrm{TE}=33$ msec; FA = 90; averages $=4$ ) coplanar to the fMRI scans to aid in registration to a standard Montreal Neurological Institute (MNI) brain, allowing fMRI analysis to take place in standard space.

\section{Functional data analysis}

All functional data analyses were performed using the Analysis Group at the Oxford Center for Functional MRI of the Brain (FMRIB) software library (FSL) tools. Skulls were first stripped automatically from each high-resolution spin echo coplanar scan using FSL Brain Extraction Tool (BET) (Jenkinson et al. 2002). FSL FMRI Expert Analysis Tool (FEAT) was then used to perform individual preprocessing and statistical analysis of each participant's fMRI scan as well as to perform group analyses. Individual participants' preprocessing included brain extraction using BET, and motion correction (Motion Correction using FMRIB's Linear Image Registration Tool [MCFLIRT]), as well as registration of each functional scan to its corresponding coplanar highresolution image using rigid body transformations, and to the MNI standard brain using nonlinear transformation. High-pass temporal filtering of $120 \mathrm{sec}$ was applied to the fMRI images, and images were spatially smoothed using a Gaussian smoothing kernel of $5 \mathrm{~mm}$.

\section{Statistical comparisons}

We performed statistical analyses on individual scans within FEAT using FMRIB's Improved Linear Model (FILM), (Woolrich et al. 2001), which applies the general linear model and uses nonparametric estimation of time series autocorrelation to remove estimated autocorrelation of noise between time points for each voxel. We first contrasted fMRI activity during retrieval of hits and correct rejections against each other and against the control condition in each condition. Thus, for the landmark recognition and spatial association conditions, we compared hits versus correct rejections, correct rejections vs. hits, hits vs. the control task, and correct rejections versus the control task. For the temporal order condition, we contrasted hits vs. incorrect responses (and the opposite contrast), hits versus the control task and incorrect vs. the control task (correct rejections could not be properly defined for the temporal order task). We used an FSL higher order mixed-effect analysis (FMRIB's Local Analysis of Mixed Effects) (Beckmann et al. 2003) to generate $Z$ statistic images $(Z>2.3$, cluster $P<0.05$, corrected) of the combined individual fMRI activation results across participants. In addition to obtaining the group results for the above comparison, we also contrasted the three different conditions against each other using a higher-order mixed-effects model. Thus, for each individual contrast, we also obtained the group activations for landmark recognition vs. (spatial association + temporal order), spatial association versus (landmark recognition + temporal order), and temporal order versus (landmark recognition + spatial association). 


\section{ROI analysis}

Because previous results demonstrated significant hippocampal, parahippocampal cortex, and retrosplenial activation during retrieval of spatial landmarks and routes (Ghaem et al. 1997; Burgess et al. 2001; Hartley et al. 2003; Janzen and van Turennout 2004), we conducted additional regions of interest analyses (ROI) on hippocampus, parahippocampal cortex, and retrosplenial cortex. We also included a perirhinal ROI mask because of the demonstrated role of perirhinal cortex in retrieving item, but not source, information (Davachi et al. 2003). Masks were created on the standard MNI brains for the hippocampus by creating anatomical masks covering the middle and posterior aspect of the hippocampus and subiculum, over posterior parahippocampal gyrus for the parahippocampal mask, and over the anterior parahippocampal gyrus lateral to entorhinal cortex for the perirhinal mask based on Duvernoy's boundaries (Duvernoy 1998). Average time courses were then extracted from the raw filtered time courses for each subject; the percent signal change was taken as the mean percent signal change over 3 and $6 \mathrm{sec}$ after the stimulus appears on the screen. All comparisons were done in a multivariate ANOVA; post-hoc tests were performed for any comparisons in ANOVAs that were significant at $P<0.05$.

\section{Results}

Behavioral data: Subjects performed above chance on all the components tasks (Fig. 1F; landmark recognition: $t_{(13)}=39$, $P<1 \times 10^{-15}$; spatial association: $t_{(13)}=4, P<0.0001$; temporal order: $\left.t_{(13)}=3.3, P<0.005\right)$. The $t$-test comparisons are based on each subject's mean performance across the entire task compared against chance (50\%). An ANOVA revealed a significant main effect between the different conditions $\left(F_{(2,41)}=57\right.$, $P<1 \times 10^{-12}$ ), with landmark recognition performed significantly better than spatial association $\left(t_{(26)}=10, P<1 \times 10^{-10}\right.$ and temporal order $\left.t_{(26)}=11, P<1 \times 10^{-11}\right)$, although there was no significant difference between spatial association and temporal order tasks $\left(t_{(26)}=.5, P=\right.$ n.s. $)$. Landmark recognition, however, was significantly different from ceiling (100\% performance; $\left.t_{(13)}=-2.2, P<0.04\right)$.

\section{Imaging data: Combined retrieval versus control}

We first identified brain areas that were commonly active across all three retrieval conditions. The combined data across the three conditions showed significant hippocampal, parahippocampal, and perirhinal cortex clusters of activation for correct retrieval of items in the experimental conditions against the control task (Fig. 2A). We could not contrast incorrect responses because subjects made too few errors in the landmark recognition condition. We also observed significant clusters activating for correct rejections vs. control in hippocampus and parahippocampal gyrus (data not shown). There were no significant differences between activations for hits and correct rejections; subsequent analyses therefore focused on comparisons of the different retrieval conditions (hits only).

\section{Region of interest (ROI) analysis in hippocampus and parahippocampal gyrus}

For each of the 14 subjects, we contrasted the magnitude of activation in the ROI analysis for hits in the landmark recognition, spatial association, and temporal order conditions in a $3 \times 2 \times 3$ ANOVA contrasting region (hippocampus vs. perirhinal cortex vs. parahippocampal cortex), laterality (right vs. left), and condition (e.g., landmark recognition vs. spatial association vs. temporal order). We observed significant effects of condition $\left(F_{(2,251)}=49, P<0.0001\right.$, region $\left(F_{(2,251)}=7, P<0.001\right)$ and significant interaction effects of region $X$ condition $\left(F_{(1,2)}=8\right.$, $P<0.0001)$ and region $\mathrm{X}$ laterality $\left(F_{(1,2)}=11, P<0.0001\right)$. No other effects reached significance. Because we found a main ef- fect of condition and region, interaction effects with region and condition, and interaction effects with region and laterality, we then proceeded to investigate differences in ROI activations using post-hoc tests on the right and left side of hippocampus and parahippocampal gyrus.

\section{Hippocampal activations}

All three behavioral conditions produced left hippocampal activations greater than baseline (Fig. $2 \mathrm{~B}$ ); we also found that both landmark recognition $\left(t_{(13)}=2.6, P<0.02\right)$ and spatial association $\left(t_{(13)}=2.3, P<0.04\right)$ conditions showed greater activation than the temporal order condition. Thus, we observed the following order of activations for left hippocampus: (spatial associations $=$ landmark recognition) $>$ temporal order. Although there were significant changes from baseline in right hippocampus for all three conditions (Fig. 2C), there was no significant difference between conditions (landmark recognition vs. spatial association, $t_{(13)}=1.5, P=$ n.s., landmark recognition vs. temporal order, $t_{(13)}=.17, P=$ n.s., spatial association vs. temporal order $t_{(13)}=1.4 P=$ n.s.). For right hippocampus, we found the following order of activations: landmark recognition = spatial association = temporal order. We also found greater left hippocampus activation during spatial association retrieval compared with right hippocampus $\left(t_{(13)}=3.3, P<0.01\right)$.

\section{Parahippocampal cortex}

For the left parahippocampal cortex (Fig. 2D), we did not observe significant signal change for the temporal order condition from baseline $\left(t_{(13)}=.74, P=\right.$ n.s. $)$, although landmark recognition and spatial association conditions showed significant levels of activation. Spatial association and landmark recognition showed greater activation than temporal order (spatial association vs. temporal order, $t_{(13)}=7.0, P<0.000005$; landmark recognition vs. temporal order, $\left.t_{(13)}=5.9, P<0.00005\right)$, and spatial association retrieval activated to a greater extent than landmark retrieval $\left(t_{(13)}=2.7, P<0.01\right)$. We observed the following order of magnitude for left parahippocampal activation: spatial association $>$ landmark recognition $>$ temporal order. In right parahippocampal cortex (Fig. 2E), we similarly found that retrieving spatial associations produced greater activation than temporal order retrieval $\left(t_{(13)}=4.7, P<0.0004\right)$; we also found that spatial association activation was significantly greater than landmark recognition activation $\left(t_{(13)}=4.2, P<0.0009\right)$. We found no difference between temporal order and landmark recognition condition activations $\left(t_{(13)}=.54, P=\right.$ n.s.). Thus, we observed the following order of activations for the right parahippocampal cortex: spatial association $>$ (landmark recognition $=$ temporal order). In contrast to the hippocampus, where spatial associations activated left greater than right hippocampus, spatial association retrieval activated right parahippocampus to a greater extent than left parahippocampus $\left(t_{(13)}=2.7, P<0.02\right)$.

\section{Perirhinal cortex}

Overall, landmark recognition resulted in greater activation bilaterally in perirhinal cortex than spatial and temporal order retrieval combined $\left(t_{(82)}=3.6, P<0.0001\right)$. The landmark recognition condition also activated left perirhinal cortex to a significantly greater extent than the spatial retrieval (left side: $t_{(13)}=3.9, P<0.001$; Fig. 2F). The landmark recognition condition was further significantly greater than the temporal order condition for both right and left perirhinal cortex (left side: $t_{(13)}=4.4, P<0.0001$; right side: $t_{(13)}=2.2, P<0.05$; Fig. $\left.2 \mathrm{G}\right)$. For left perirhinal cortex, landmark $>$ (temporal order $=$ spatial associations). For right perirhinal cortex, (landmark recognition $=$ spatial associations) $>$ temporal order. 


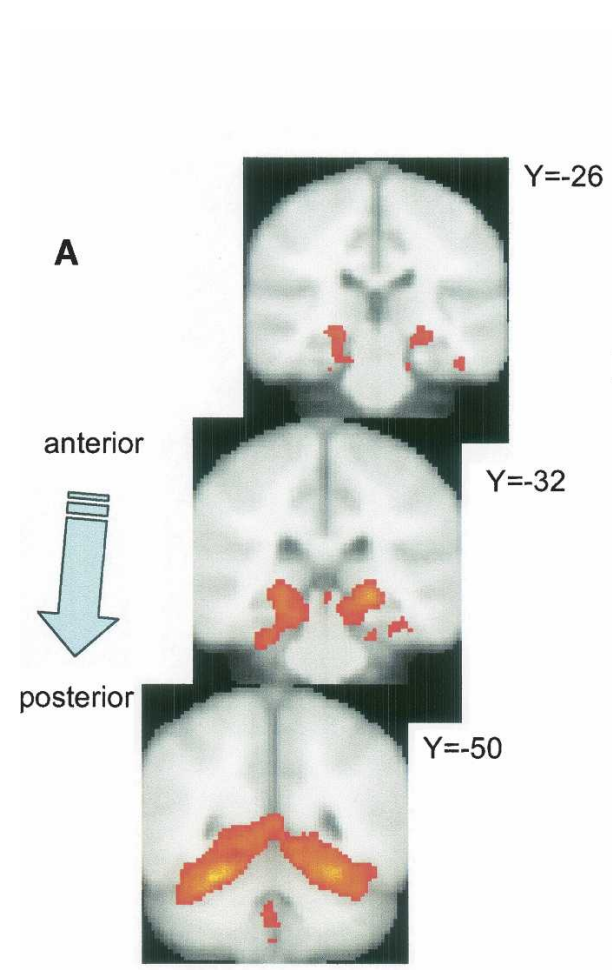

2.3

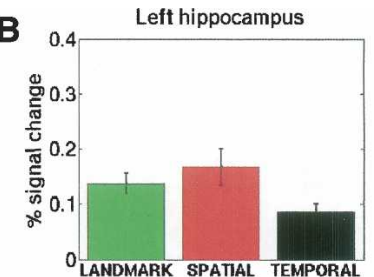

D
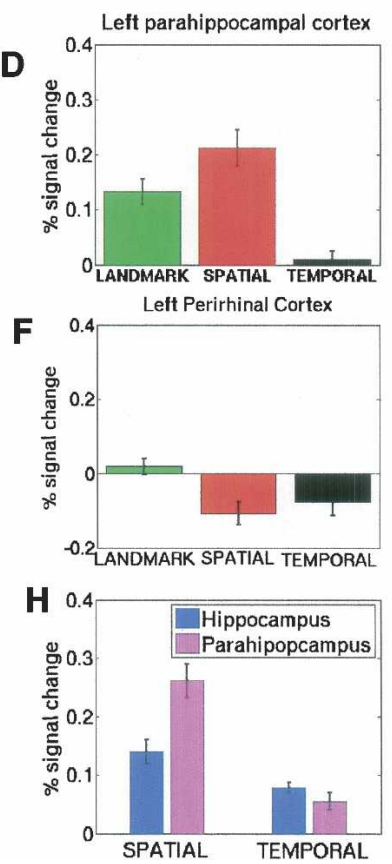
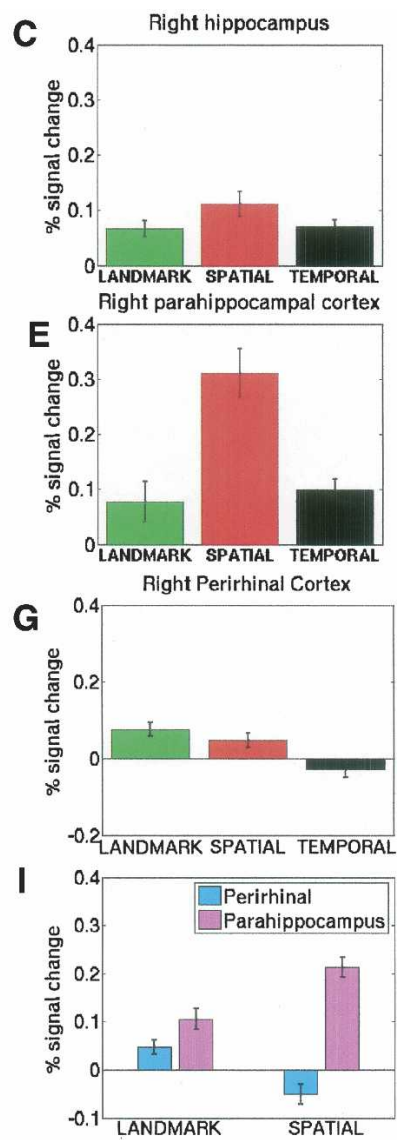

Figure 2. Activations in the hippocampus and parahippocampal cortex during retrieval of recently learned spatial information. (A) Group mean cluster activations in standard MNI space for 14 subjects during retrieval of landmarks, passenger-store associations (spatial association condition), and orderings of passengers (temporal order condition). We observed significant bilateral hippocampal, parahippocampal, and perirhinal cortex activations. Numbers next to slices indicate MNI values in millimeters of slice locations. Activations were thresholded at $Z=2.3$. ROI analysis for these regions shown in $B-G$. (B) In left hippocampus, we observed greater activations during landmark recognition and spatial association conditions compared with the temporal order condition. (C) In right hippocampus, we did not find differences between conditions. However, all three conditions activated hippocampus bilaterally. $(D)$ Left parahippocampal cortex showed greater activation during spatial association retrieval than the other two conditions; landmark recognition activated the left parahippocampal cortex to a greater extent than temporal order retrieval. (E) Right parahippocampal activation was also higher during spatial association retrieval than the other two conditions. $(F)$ Left perirhinal activation was higher during landmark retrieval than spatial and temporal retrieval. (G) Right perirhinal activity was higher during landmark retrieval compared with temporal order retrieval. $(H)$ We observed a significant dissociation between hippocampal and parahippocampal activations during spatial and temporal order retrieval; spatial retrieval activated parahippocampus to a greater extent than hippocampus, and temporal order retrieval activated hippocampus to a greater extent than parahippocampus. (I) We also found a significant dissociation between parahippocampal cortex and perirhinal cortex; spatial retrieval led to greater activation in parahippocampal cortex compared with landmark retrieval, while the opposite trend (greater activation during landmark than spatial retrieval) was observed in perirhinal cortex.

\section{Comparing hippocampus, parahippocampal, and perirhinal cortex activations}

To better understand the region $\mathrm{X}$ condition interaction effect we observed, and to test our original hypothesis regarding differential roles of hippocampus and parahippocampal cortex in temporal versus spatial memory, we conducted a separate ANOVA only on activations in hippocampus and parahippocampal cortex during spatial association and temporal order conditions. We found a significant interaction effect between parahippocampal cortex and hippocampal activations (Fig. 2H): Hippocampal activation was greater during temporal order retrieval than parahippocampal cortex, while parahippocampal activation was greater during the spatial association condition compared with hippocampus $(2 \times 2$ region $\mathrm{X}$ condition ANOVA, region $\mathrm{X}$ condition interaction, $P<0.01)$. To test our hypothesis regarding item and source retrieval differences between parahippocampal and perirhinal cortex, we also conducted an additional ANOVA comparing parahippocampal cortex activation and perirhinal cortex activation during landmark and spatial recognition conditions (Fig. 2I). We again found a significant region $X$ condition interaction $(2 \times 2$ region $X$ condition ANOVA, region $X$ condition interaction, $P<0.01)$.

\section{Group analysis: Extra-hippocampal activations}

Prior studies also implicate extra-hippocampal structures in spatial navigation and spatial retrieval, particularly retrosplenial cortex (Shelton and Gabrieli 2002; Hartley et al. 2003; Rosenbaum et al. 2004; Wolbers and Buchel 2005). In line with these studies, we found significant retrosplenial activation generally during retrieval (Fig. 3A). Because of our a priori hypothesis regarding the presence of retrosplenial activation during spatial retrieval, we conducted an additional ROI analysis on the retrosplenial cortex based on the anatomical definition provided by Maguire (2001), comprised of Broadman areas 29 and 30. While the landmark condition activated to a significantly greater extent than the temporal order condition $\left(t_{(13)}=2.4, P<0.03\right)$, we did not find 


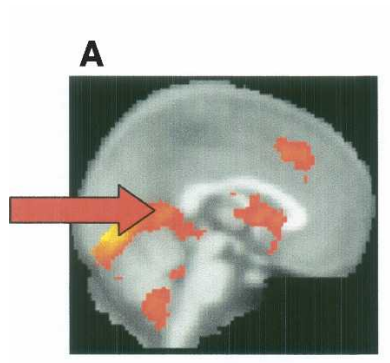

2.3
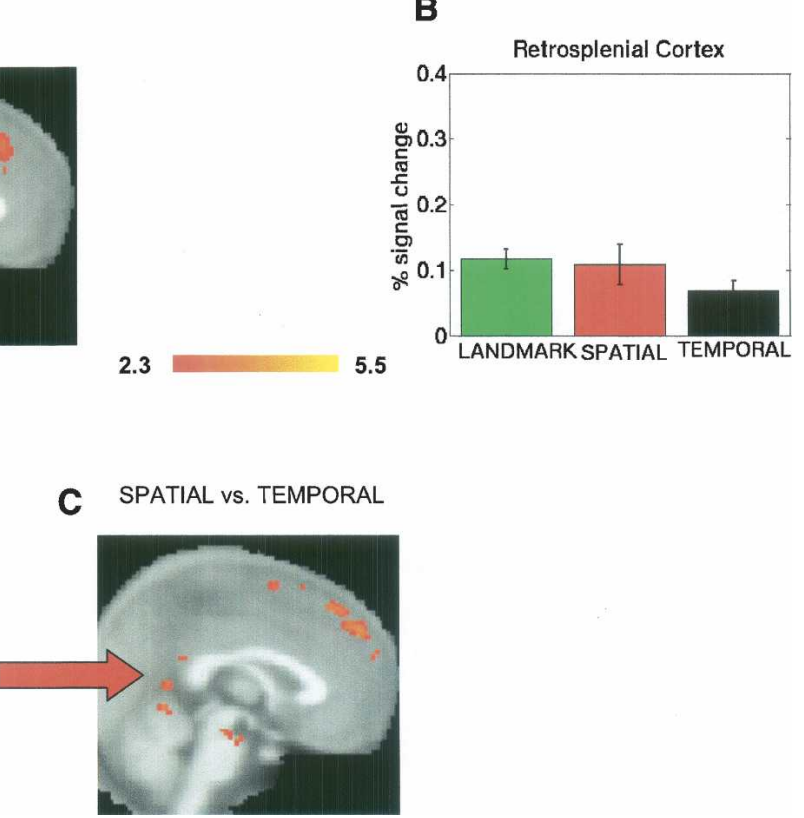

Figure 3. Extra-hippocampal activations and retrosplenial region of interest analysis. (A) Outside of the hippocampus, significant activations in retrosplenial cortex, prefrontal cortex, and cingulate are readily identifiable in the mean across all three retrieval conditions. (B) ROI of retrosplenial cortex indicated overall significantly more activation during landmark retrieval and spatial association retrieval (combined) compared with temporal order; the contrast of spatial association vs. temporal order failed to reach significance. (C) In a group analysis, comparison of the spatial retrieval condition against temporal order retrieval, distinct clusters of activation were present in retrosplenial cortex. Activations were thresholded at $Z=2.3$. Significant retrosplenial clusters of activation were also present when we contrasted landmark recognition against temporal order retrieval; no other contrasts showed significant retrosplenial activations.

greater activation during the spatial association condition compared with the temporal order condition (Fig. 3B). Thus, for retrosplenial cortex, we found landmark recognition $>$ temporal order. Landmark and spatial conditions together, however, were significantly greater than the temporal order condition $\left(t_{(40)}=2.3, P<0.03\right)$. An additional mixed-effects analysis comparing spatial association retrieval against the temporal order condition also revealed significant, yet localized, clusters in the retrosplenial cortex (Fig. 3C). Consistent with the ROI analysis, we also found significant clusters of activations in retrosplenial cortex when we contrasted landmark with temporal order retrieval; we did not find significant retrosplenial activation during any other comparisons. These data suggest that our ROI analysis did not locate the spatial retrieval activation in retrosplenial cortex compared with temporal order, because the clusters were fairly small and localized. We suggest that the order of activation for retrosplenial cortex instead be (landmark recognition $=$ spatial associations) $>$ temporal order.

Table 1 presents a comprehensive list of brain regions active during retrieval. These regions included activations in prefrontal cortex, cingulate cortex, lingual, and fusiform gyri. The most robust activations during retrieval were present bilaterally in fusiform and lingual gyri. To identify extra-hippocampal activations that were unique to each retrieval condition, we conducted an additional comparison of each condition against the other two conditions using a $t$-test $(P$ corrected). Thus, we contrasted landmark recognition against spatial association and temporal order, spatial association against landmark recognition and temporal order, and temporal order against landmark recognition and spatial association. Consistent with recent studies on route sequence learning (Hartley et al. 2003), the temporal order condition uniquely activated regions in the striatum, such as the caudate nucleus (Table 2). We also found robust activations in middle frontal gyrus (MFG: prefrontal cortex) during spatial and temporal but not landmark retrieval, consistent with previous studies on retrieval of information from recently experienced VR environments (King et al. 2005). In fact, the landmark recognition condition showed no areas that were active above the levels of the temporal order and the spatial association conditions (e.g., Table 2). We conducted additional analyses comparing each condition against the other (e.g., 6-way comparison of landmark vs. spatial, spatial vs. landmark, etc.). Because these analyses did not reveal any additional information not already contained in the 3-way comparison or ROI analysis, we do not present it or discuss it further in this work.

\section{Discussion}

By having subjects freely explore a virtual environment and then retrieve landmark, spatial association, and temporal order information while undergoing fMRI imaging, we compared brain regions involved in the retrieval of different components of episodic memory. We observed significant activations in hippocampus and parahippocampal gyrus during landmark, spatial association, and temporal order retrieval; we did not find activation differences for retrieving familiar compared with novel landmarks and associations. These data argue for the involvement of MTL regions in the process of retrieval of episodic information from memory. Our data further argue for the involvement of specific regions within medial temporal lobe for retrieving spatial and temporal order information. Parahippocampal cortex activated bilaterally during retrieval of spatial associations compared with hippocampus, while hippocampus activated bilaterally during retrieval of temporal order information

Table 1. Brain regions active during retrieval

\begin{tabular}{|c|c|c|c|c|c|}
\hline \multicolumn{2}{|l|}{ Condition } & \multicolumn{4}{|c|}{ Group mean vs. control } \\
\hline Region & & $x$ & $y$ & $z$ & $Z$ \\
\hline \multicolumn{6}{|l|}{ 1. Medial temporal } \\
\hline \multirow{2}{*}{ Hippocampus } & $\mathrm{L}$ & 24 & -28 & 10 & 4.6 \\
\hline & $\mathrm{R}$ & -24 & -28 & 10 & 2.8 \\
\hline \multirow[t]{2}{*}{ Parahippocampal gyrus } & $\mathrm{L}$ & -24 & -18 & -38 & 3.2 \\
\hline & $\mathrm{R}$ & 24 & -34 & -20 & 4.4 \\
\hline \multirow[t]{2}{*}{ 2. Lingual gyrus } & $\mathrm{L}$ & -12 & -84 & -14 & 8.8 \\
\hline & $\mathrm{R}$ & 10 & -82 & -12 & 8.2 \\
\hline \multirow[t]{2}{*}{ 3. Fusiform gyrus } & $\mathrm{L}$ & -26 & -74 & -18 & 8.3 \\
\hline & $\mathrm{R}$ & 24 & -82 & -22 & 8.0 \\
\hline \multirow[t]{2}{*}{ 4. Cingulate gyrus } & $\mathrm{L}$ & -8 & 16 & 46 & 4.1 \\
\hline & $\mathrm{R}$ & 8 & 30 & 26 & 3.3 \\
\hline 5. Cerebellum (medial) & $\mathrm{L}$ & 0 & -56 & 46 & 3.9 \\
\hline \multicolumn{6}{|l|}{ 6. Prefrontal } \\
\hline \multirow[t]{2}{*}{ IFG } & $\mathrm{R}$ & 46 & 26 & 20 & 5.2 \\
\hline & $\mathrm{L}$ & -38 & 24 & -2 & 4.2 \\
\hline \multirow[t]{2}{*}{ MFG } & $\mathrm{R}$ & 58 & 26 & 28 & 4.6 \\
\hline & $\mathrm{L}$ & -2 & 32 & 40 & 4.0 \\
\hline
\end{tabular}

$x, y$, and $z$ reflect positions in $\mathrm{MNI}$ coordinate space; $Z$ reflects maximum $Z$ value in the cluster. 
Table 2. Brain regions active during item, spatial, and temporal episodic memory retrieval

\begin{tabular}{|c|c|c|c|c|c|c|c|c|c|c|c|c|c|}
\hline \multirow{2}{*}{$\begin{array}{c}\text { Condition } \\
\text { Region }\end{array}$} & & \multicolumn{4}{|c|}{$\begin{array}{l}\text { Landmark vs. } \\
\text { Spatial + Temporal }\end{array}$} & \multicolumn{4}{|c|}{$\begin{array}{c}\text { Spatial vs. } \\
\text { Landmark + Temporal }\end{array}$} & \multicolumn{4}{|c|}{$\begin{array}{c}\text { Temporal vs. } \\
\text { Spatial + Landmark }\end{array}$} \\
\hline & & $x$ & $y$ & $z$ & $z$ & $x$ & $y$ & $z$ & $z$ & $x$ & $y$ & $z$ & $z$ \\
\hline 1. MTL (parahippocampal gyrus) & $\mathrm{R}$ & & & & & 20 & -26 & -26 & 2.7 & & & & \\
\hline 2. Fusiform gyrus & $\mathrm{R}$ & & & & & 40 & -44 & -26 & 3.1 & -38 & -52 & -18 & 4.3 \\
\hline 3. Cingulate (anterior) & L & & & & & & & & & -8 & 20 & 42 & 3.5 \\
\hline 4. Motor (precentral gyrus) & $\mathrm{L}$ & & & & & -50 & 2 & 48 & 3.2 & & & & \\
\hline 5. Cerebellum (medial) & L & & & & & -4 & -68 & -48 & 3.4 & & & & \\
\hline \multicolumn{14}{|l|}{ 6. Prefrontal } \\
\hline SFG & $\mathrm{L}$ & & & & & -14 & 36 & 54 & 3.7 & & & & \\
\hline MFG & $\mathrm{R}$ & & & & & 2 & 48 & 48 & 3.2 & 54 & 22 & 28 & 3.8 \\
\hline IFG & L & & & & & -46 & 24 & -2 & 3.9 & -32 & 26 & 0 & 3.0 \\
\hline & $\mathrm{R}$ & & & & & & & & & 48 & 28 & 20 & 4.0 \\
\hline \multicolumn{14}{|l|}{ 7. Temporal Cortex } \\
\hline ITG & $\mathrm{R}$ & & & & & & & & & 56 & -72 & -4 & 5.6 \\
\hline MTG & $\mathrm{R}$ & & & & & & & & & 50 & -66 & 6 & 5.0 \\
\hline Insula & & & & & & & & & & -36 & 18 & 0 & 3.3 \\
\hline \multicolumn{14}{|l|}{ 8. Occipital Cortex } \\
\hline \multirow[t]{2}{*}{ Medial } & $\mathrm{R}$ & & & & & & & & & 44 & -72 & -2 & 4.8 \\
\hline & L & & & & & & & & & -48 & -80 & 0 & 4.5 \\
\hline \multirow[t]{2}{*}{ Inferior } & $\mathrm{R}$ & & & & & & & & & 48 & -80 & -10 & 4.7 \\
\hline & $\mathrm{L}$ & & & & & & & & & -42 & -84 & -6 & 4.5 \\
\hline \multicolumn{14}{|l|}{ 9. Subcortical } \\
\hline Substantia nigra & $\mathrm{R}$ & & & & & 4 & 18 & -22 & 3.2 & & & & \\
\hline Putamen & L & & & & & & & & & -16 & 12 & -2 & 2.9 \\
\hline Caudate & & & & & & & & & & -6 & 16 & 38 & 3.4 \\
\hline Globus pallidus & $\mathrm{L}$ & & & & & & & & & -12 & 4 & -2 & 3.0 \\
\hline
\end{tabular}

$x, y$, and $z$ reflect positions in MNI coordinate space; $Z$ reflects maximum $Z$ value in the cluster.

compared with parahippocampal cortex. We also found differences in the involvement of right and left hippocampus in retrieval of spatial associations: We found greater activation in left hippocampus compared with right hippocampus, and greater right parahippocampal activation compared with left parahippocampal activation. There were also activation differences between parahippocampal and perirhinal cortex. We found significantly greater parahippocampal cortex activation bilaterally during spatial association retrieval compared with landmark retrieval, while perirhinal cortex showed greater activation during landmark retrieval compared with spatial association retrieval. Finally, extra-hippocampal brain regions differentially activated for spatial compared with temporal order memory. Retrosplenial cortex activated during spatial association retrieval and landmark recognition compared with temporal order retrieval. Also, striatum activated to a greater extent during the temporal order condition compared with the other two conditions. We discuss below the functional relevance of these activations and how these results relate to our hypothesis stated in the introduction.

\section{Hippocampal and parahippocampal gyrus activations}

The primary focus of this study was to compare spatial and temporal judgments following a recently experienced episode to attempt to resolve competing hypotheses on the role of the parahippocampal cortex and hippocampus in spatial and temporal order memory judgments (Tulving 1993; Burgess et al. 2002; Eichenbaum 2004). Important to this comparison, subjects performed equally well, but well above chance, on both spatial and temporal order judgments (Fig. 1F). In attempting to equate the difficulty of spatial and temporal order retrieval, we designed the spatial association condition so that it involved rapid judgments about whether a passenger went to a specific store or not. It could be argued that subjects may have learned the spatial associations task by simply pairing specific passengers with specific stores based on nonspatial strategies. Two lines of reasoning argue against this. First, in a prior study using yellowcab2, in which subjects picked up passengers and delivered them to stores, subjects quickly learned the locations of stores over trials by gradually optimizing their paths from passengers to stores-even though subjects were not explicitly told to do so (Newman et al. 2006). Second, the patterns of activations we observed also argue against subjects using a nonspatial strategy to retrieve passengerstore associations. Consistent with previous studies (Janzen and van Turennout 2004), we found the greatest parahippocampal activation when subjects retrieved landmarks in a spatially relevant manner compared with simply viewing the landmark alone during the landmark recognition task (Fig. 2D,E). Were subjects using a nonspatial strategy, the predicted activation patterns for parahippocampal cortex instead would be equivalent activation in all three conditions, or at least equivalent activation during the landmark and spatial-association condition.

A possible difficulty in comparing the spatial associations condition with the temporal-order condition relates to the fact that subjects saw a passenger and a store on the screen during spatial association retrieval and two passengers during temporalorder retrieval. During the task, however, subjects were explicitly instructed to make judgments about the relation between the stimuli, and were reminded to do so during stimulus presentation by lines of text that appeared continuously below the stimuli (e.g., "Which store did you take me to?" and "Whom did you meet first?"; see Fig. 1D,E). Thus, the main focus of the subject was remembering the trajectory the passenger traveled to the store or the order the passenger came relative to the other passenger shown and not specific stimulus features. Although stronger parahippocampal activations are typically observed for spatial scenes compared with people or other objects (Epstein and Kanwisher 1998), we note that the stores themselves were not spatial scenes, but rather objects that served as landmarks during the game. Finally, we did not observe differences for retrieving novel vs. familiar stores or passenger-store pairings. These results argue that the differences in activations we observed in the three conditions derived primarily from judgments subjects made about the different stimuli rather than the stimuli themselves. 
A final issue we address concerns comparing the landmark recognition condition against the associative recognition conditions. Subjects performed significantly better on this task compared with the associative recognitions, in part because familiarity-based recognition judgments of picture stimuli are typically easier for subjects than associative-based recognition judgments. For example, in a recent study by Holdstock et al. (2005), subjects performed significantly better on familiarity-based recognition of pictures compared with spatial and temporal tasks. If task difficulty were to have an effect on our results, we would expect significantly less regional activation during landmark recognition compared with associative recognition. The complex pattern of activations we observed within in the MTL-decreasing perirhinal activation from landmark to associations tasks, yet increasing parahippocampal activations and equivalence in activation for right hippocampus-argues against this explanation. The dissociations we observed between regions and conditions addresses a concern mentioned in the Materials and Methods with having landmark recognition precede associative recognition (although associative recognition conditions were counterbalanced). Were this to have significantly affected our results, we would have expected decreasing activation in all regions over conditions due to adaptation-based processes, instead of the findings we report.

Similar to our study, two fMRI studies by Burgess et al. (2001) and King et al. (2005) also investigated activations during memory retrieval following virtual navigation in the hippocampal area. In the two studies, subjects moved through a virtual environment to different spatial locations, at which they met specific people who held certain objects. Subjects then made decisions in the scanner about whether they had encountered a certain object (1) in that place, (2) with that person, or (3) with an additional object. As a control, subjects also made judgments about the width or brightness of objects. Burgess et al. (2001) found primarily left hippocampal activation only in the place vs. control comparison, arguing for the involvement of the left hippocampus in the processing and retrieval of spatialcontextual information. In our study, we similarly found greater left hippocampal activation during spatial association retrieval compared with right hippocampus. Indeed, the $Z$ scores we observed for right and left hippocampus (right: $Z=2.8$; left: $Z=4.6$ ) are generally comparable to those observed in the Burgess et al. (2001) study (Burgess et al. report $Z=3.6$ only for left hippocampus).

There are, however, several notable differences between our studies that make more in-depth comparisons difficult. One important difference is that our subjects navigated the spatial environment freely compared with the Burgess et al. (2001) and King et al. (2005) studies, in which subjects were guided with arrows to specific locations. Arguably, our paradigm allowed subjects to encode information in a fashion similar to how we might encode episodic information in the natural world compared with the Burgess et al. and King et al. studies in which the information experienced was constrained by the details of the experiment. A second important difference between our study and that of the previous two studies is the control conditions. Burgess et al. and King et al. used a control condition that required subjects to make perceptual judgments about the pictures of objects or places they visited-presumably leading to little hippocampal activation. Our study, in contrast, used a low-level perceptual task (determining whether a number was odd or even)—shown in a previous study by Stark and Squire (2001) to provide minimal hippocampal activation compared with picture viewing. Our control task, in fact, may account for the slightly greater $Z$ values and percent signal changes we observed when we compared retrieval conditions against the control condition (this study, Table
1 and Fig. 2; Burgess et al. 2001, Table 1 and Fig. 4). A third difference between our studies is that the memory component of our study required subjects to make recognition judgmentsparticularly in the landmark and spatial conditions-while the Burgess et al. (2001) and King et al. (2005) studies required subjects to make forced-choice judgments about which objects came when. Although the Burgess et al. and King et al. memory condition allowed subjects to look at the same stimuli but make different types of judgments about them, a manipulation not possible in our study, our paradigm permitted comparison with other studies that have looked separately at item and source memory retrieval. A final difference between our two studies is that our study looked at temporal order retrieval in addition to spatial and object memory.

Because our study allowed us to directly compare simple item recognition with recognition requiring recovery of spatial and temporal source information, we believe our results demonstrate several important aspects of the brain networks underlying episodic retrieval. First of all, our data demonstrate the general involvement of the hippocampus and parahippocampal cortex in retrieval of recently experienced items, spatial associations, and temporally sequenced events in episodic memory. Our results therefore suggest a general role for the hippocampus in retrieval of episodic memories (Tulving 1993) - at least those acquired from a recently experienced spatial environment. We predicted, however, greater parahippocampal cortex than hippocampal activation during landmark retrieval based on the fact that the parahippocampal cortex typically shows stronger activation during familiarity-based recognition than the hippocampus (Epstein and Kanwisher 1998). The fact that we saw similar degrees of hippocampal and parahippocampal cortex activation during landmark recognition-contrary to our expectationsuggests that the hippocampus and parahippocampal cortex were recruited to comparable degrees during the landmark recognition task. In contrast to hippocampus and parahippocampal cortex, perirhinal cortex showed greater activation during landmark compared with spatial and temporal retrieval. We also noted dissociation between perirhinal and parahippocampal cortical activations-perirhinal cortex activated more strongly during landmark than spatial retrieval, while parahippocampal cortex activated more strongly during spatial than landmark retrieval. Together, our data suggest a more specific role for the perirhinal cortex in retrieval of item, and not source information, from recently experienced events (Davachi et al. 2003).

Compared with hippocampus, parahippocampal cortex activated to a greater extent during spatial association retrieval than temporal order retrieval (e.g., Fig. 2H); hippocampus, in contrast, activated more strongly than parahippocampal gyrus cortex during temporal order retrieval compared with spatial retrieval. Our dissociation of hippocampal and parahippocampal activations during temporal order and spatial association retrieval suggests the continued involvement of the parahippocampal cortex in spatial memory, but suggests that it plays less of a role in temporal order memory, a key component of episodic memory (Tulving 1993). Our data also argue for a greater hippocampal role in temporal order memory than the parahippocampal cortex; previous studies have also reported hippocampal activation during temporal order retrieval (Rekkas et al. 2005). Our findings thus provide an important prediction for patients with medial temporal lobe lesions: Patients with lesions restricted to hippocampus should show profound temporal order memory deficits, but lesions that extend into the parahippocampal cortex should not further impair temporal order memory.

A final activation difference of note in the MTL is that spatial association retrieval led to greater left hippocampal activation compared with temporal order retrieval; we did not observe 
a difference between the three conditions for right hippocampal retrieval. These data suggest that the left hippocampus may play a greater role in spatial retrieval than temporal order retrieval. Consistent with out findings, Spiers et al. (2001) found greater impairment during spatial retrieval for left hippocampal damaged patients compared with right hippocampally damaged patients. In the Spiers et al. study, subjects navigated a virtual environment; following navigation, patients performed several tests of episodic memory, recalling people, objects, and the order of events experienced during virtual navigation. Left hippocampally damaged patients showed the greatest impairments during retrieval of these episodic experiences compared with right hippocampally damaged patients, who had impairments during navigation and in map drawing. Together with our findings, these data support the idea that the left hippocampus may play a greater role in spatial retrieval than right hippocampus.

\section{Extra-hippocampal activations: Retrosplenial cortex}

Retrosplenial cortex showed significant activation when subjects retrieved spatial associations and landmarks compared with retrieval of temporal order information. Previous findings suggested the importance in general of the posterior parietal cortex in retrieval of episodic memories (Shallice et al. 1994; Nyberg et al. 1996). Fujii et al. (2004), however, found the greatest activations in retrosplenial cortex when subjects retrieved spatial episodic information. In the Fujii et al. (2004) study, subjects experienced several different experimentally induced real world events and then answered questions about whether the events happened or not, where they occurred, and the order in which they happened. Retrosplenial cortex activation was greater during retrieval of spatial information compared with remembering the order of events or during event recognition. Previous fMRI studies further suggest retrosplenial recruitment may be particularly salient during retrieval of topographic spatial information compared with retrieval of route information (Shelton and Gabrieli 2002; Wolbers and Buchel 2005). For example, Wolbers and Buchel (2005) found that retrosplenial activation correlated with subjects successfully drawing survey maps of environments learned over multiple scanning sessions. Based on these findings, we tentatively suggest that the retrosplenial cortex activations observed in our study, most pronounced during spatial association and landmark retrieval compared with temporal order retrieval, reflect the accessing of topographic spatial representations of the virtual environment subjects explored to aid in retrieval of which passengers went to which stores and which stores were visited.

\section{Prefrontal cortex and other extra-hippocampal activations}

We found that medial frontal gyrus (MFG) and fusiform cortex were active in the spatial association and temporal order tasks when we contrasted against the landmark recognition task (Table 2). These findings suggest that both spatial association and temporal order conditions involved recruitment of these two structures. Leung et al. (2002) found that MFG activation increased with increasing memory loads, showing little activation during delay periods requiring maintenance of three items, but robust activation during delay periods requiring maintenance of five items. In our study, the spatial association and temporal order conditions required holding one item in memory while comparing it against the other item (either a store or a passenger in our case). We therefore attribute the prefrontal activations in our study to the increased memory load involved in the spatial associations and temporal order conditions. King et al. (2005) found less prefrontal cortex activation when subjects had to recall from a larger pool of places, objects, and people compared with the smaller pool used in Burgess et al. (2001), likely due to the lower contextual interference present with a greater number of unique episodes. Indeed, our activations $(Z=3.2)$ were closer in line with those of King et al. $(Z=3.5)$ than Burgess et al. $(Z=4.4)$; correspondingly, we had comparable numbers of unique events to King et al. (2005). Based on our convergence with King et al. as well as the previously demonstrated role of MFG in short-term memory, we suggest that our activations in prefrontal cortex represent the cognitive resources involved in matching specific passengers with stores and comparing different passengers against each other in time.

Fusiform cortex, which showed the strongest magnitude activation during retrieval $(Z=8.3)$ of any region, displayed a similar pattern to prefrontal cortex of activating during temporal order and spatial association conditions, but not during landmark recognition (Table 2 ). We similarly attribute the greater activation there during spatial and temporal retrieval to the presence of two objects in memory compared with one, as two objects would require greater processing demands than simply recognizing single objects. Based on the previously demonstrated role of the fusiform gyrus in object recognition (Grill-Spector et al. 2006), we suggest that the fusiform activations observed in this study represent the processing of several objects simultaneously. We also note that the lingual gyrus was active generally during retrieval (Table 1) but were not active during specific contrasts (Table 2). These data suggest that this structure plays a somewhat more general role in object processing during retrieval.

\section{Striatal activations}

A final important difference we observed between conditions was activation of the striatum in the temporal order condition but not in the spatial association or landmark recognition condition (Table 2). The striatum activates during motor sequence learning, both while subjects have explicit and implicit knowledge of a sequence (Willingham et al. 2002; Destrebecqz et al. 2005). Studies by Reeves et al. (2005) and Hartley et al. (2003) further showed striatal activity during retrieval of sequentially learned spatial information. These findings suggest that the striatal activation in our study during temporal order retrieval is likely related to the retrieval of sequences.

\section{Conclusion}

Our results support the involvement of several different brain networks in the retrieval of previously learned episodic and spatial information. The hippocampus and parahippocampal cortex activated during retrieval in all three conditions, although parahippocampal activations were particularly pronounced during spatial association retrieval. Our data support a role for the hippocampus and parahippocampal gyrus in representation of item, spatial, and temporal context in episodic memory. We also report a novel dissociation between hippocampal and parahippocampal involvement, with the hippocampus more active during correct retrieval of temporal order information compared with the parahippocampal cortex, and parahippocampal cortex more active during correct retrieval of spatial associations compared with the hippocampus. These results support a role for the two structures in spatial and temporal source memory retrieval, respectively. Compared with parahippocampal cortex, perirhinal cortex showed greater activation during landmark retrieval compared with spatial retrieval, suggesting a more limited role in item representation. Retrosplenial activation was similarly greater during spatial association and landmark retrieval, and may relate to the use of topographic maps when subjects successfully remembered which passengers went to which stores. Temporal order retrieval, in contrast, led to greater striatal activation, relating to its previous established role in sequence learning and retrieval. Our re- 
sults suggest that distinct networks of brain regions are recruited when retrieving the spatial and temporal context of newly learned episodes; these activations in turn relate to the behavioral function these regions are primarily involved in, as suggested by our results and previous findings discussed in this study.

\section{Acknowledgments}

We thank Barbara Knowlton, Itzhak Fried, and Indre Viskontas for helpful discussions and comments on this manuscript and support from NINDS F32 NS50067-01A1 to A.E. We also thank M.J. Kahana for sharing and support of the virtual navigation task "yellowcab," Josh Jacobs and Matt Mollison for technical support of yellowcab, and NIH grant MH61975 to M.J. Kahana for supporting the development of yellowcab. For their generous support, the authors thank the Brain Mapping Medical Research Organization, Brain Mapping Support Foundation, PiersonLovelace Foundation, The Ahmanson Foundation, William M. and Linda R. Dietel Philanthropic Fund at the Northern Piedmont Community Foundation, Tamkin Foundation, Jennifer JonesSimon Foundation, Capital Group Companies Charitable Foundation, Robson Family, and Northstar Fund. The project described was also supported by NIH grants RR12169, RR13642, and RR00865.

\section{References}

Aggleton, J.P. and Brown, M.W. 2006. Interleaving brain systems for episodic and recognition memory. Trends Cogn. Sci. 10: 455-463.

Aguirre, G.K., Detre, J.A., Alsop, D.C., and D'Esposito, M. 1996. The parahippocampus subserves topographical learning in man. Cereb. Cortex 6: 823-829.

Beckmann, C.F., Jenkinson, M., and Smith, S.M. 2003. General multilevel linear modeling for group analysis in FMRI. Neuroimage 20: $1052-1063$.

Bohbot, V.D., Kalina, M., Stepankova, K., Spackova, N., Petrides, M., and Nadel, L. 1998. Spatial memory deficits in patients with lesions to the right hippocampus and to the right parahippocampal cortex. Neuropsychologia 36: 1217-1238.

Burgess, N., Maguire, E.A., Spiers, H.J., and O'Keefe, J. 2001. A temporoparietal and prefrontal network for retrieving the spatial context of lifelike events. Neuroimage 14: 439-453

Burgess, N., Maguire, E.A., and O'Keefe, J. 2002. The human hippocampus and spatial and episodic memory. Neuron 35: 625-641.

Davachi, L., Mitchell, J.P., and Wagner, A.D. 2003. Multiple routes to memory: Distinct medial temporal lobe processes build item and source memories. Proc. Natl. Acad. Sci. 100: 2157-2162.

Destrebecqz, A., Peigneux, P., Laureys, S., Degueldre, C., Del Fiore, G., Aerts, J., Luxen, A., Van Der Linden, M., Cleeremans, A., and Maquet, P. 2005. The neural correlates of implicit and explicit sequence learning: Interacting networks revealed by the process dissociation procedure. Learn. Mem. 12: 480-490.

Duvernoy, H.M. 1998. The human hippocampus: Functional anatomy, vascularization, and serial sections with MRI. Springer, Berlin, Germany.

Eichenbaum, H. 2004. Hippocampus: Cognitive processes and neural representations that underlie declarative memory. Neuron 44: 109-120.

Ekstrom, A.D., Kahana, M.J., Caplan, J.B., Fields, T.A., Isham, E.A., Newman, E.L., and Fried, I. 2003. Cellular networks underlying human spatial navigation. Nature 425: 184-188.

Ekstrom, A.D., Caplan, J.B., Ho, E., Shattuck, K., Fried, I., and Kahana, M.J. 2005. Human hippocampal $\theta$ activity during virtual navigation. Hippocampus 15: 881-889.

Epstein, R. and Kanwisher, N. 1998. A cortical representation of the local visual environment. Nature 392: 598-601.

Fujii, T., Suzuki, M., Okuda, J., Ohtake, H., Tanji, K., Yamaguchi, K., Itoh, M., and Yamadori, A. 2004. Neural correlates of context memory with real-world events. Neuroimage 21: 1596-1603.

Ghaem, O., Mellet, E., Crivello, F., Tzourio, N., Mazoyer, B., Berthoz, A., and Denis, M. 1997. Mental navigation along memorized routes activates the hippocampus, precuneus, and insula. Neuroreport 8: 739-744.

Grill-Spector, K., Sayres, R., and Ress, D. 2006. High-resolution imaging reveals highly selective nonface clusters in the fusiform face area. Nat. Neurosci. 9: 1177-1185.

Hartley, T., Maguire, E.A., Spiers, H.J., and Burgess, N. 2003. The well-worn route and the path less traveled: Distinct neural bases of route following and wayfinding in humans. Neuron 37: 877-888.

Holdstock, J.S., Mayes, A.R., Gong, Q.Y., Roberts, N., and Kapur, N. 2005. Item recognition is less impaired than recall and associative recognition in a patient with selective hippocampal damage. Hippocampus 15: 203-215.

Janzen, G. and van Turennout, M. 2004. Selective neural representation of objects relevant for navigation. Nat. Neurosci. 7: 673-677.

Jenkinson, M., Bannister, P., Brady, M., and Smith, S. 2002. Improved optimization for the robust and accurate linear registration and motion correction of brain images. Neuroimage 17: 825-841.

King, J.A., Hartley, T., Spiers, H.J., Maguire, E.A., and Burgess, N. 2005. Anterior prefrontal involvement in episodic retrieval reflects contextual interference. Neuroimage 28: 256-267.

Leung, H.C., Gore, J.C., and Goldman-Rakic, P.S. 2002. Sustained mnemonic response in the human middle frontal gyrus during on-line storage of spatial memoranda. J. Cogn. Neurosci. 14: 659-671.

Maguire, E.A. 2001. The retrosplenial contribution to human navigation: A review of lesion and neuroimaging findings. Scand. J. Psychol. 42: 225-238.

Newman, E.L., Caplan, J.B., Kirschen, M.P., Korolev, I.O., Sekuler, R., and Kahana, M.J. 2006. Learning your way around town: How virtual taxicab drivers learn to use both layout and landmark information. Cognition 104: 231-253.

Nyberg, L., McIntosh, A.R., Cabeza, R., Habib, R., Houle, S., and Tulving, E. 1996. General and specific brain regions involved in encoding and retrieval of events: What, where, and when. Proc. Natl. Acad. Sci. 93: 11280-11285.

Reeves, S.J., Grasby, P.M., Howard, R.J., Bantick, R.A., Asselin, M.C., and Mehta, M.A. 2005. A positron emission tomography (PET) investigation of the role of striatal dopamine (D2) receptor availability in spatial cognition. Neuroimage 28: 216-226.

Rekkas, P.V., Westerveld, M., Skudlarski, P., Zumer, J., Pugh, K., Spencer, D.D., and Constable, R.T. 2005. Neural correlates of temporal-order judgments versus those of spatial-location: Deactivation of hippocampus may facilitate spatial performance. Brain Cogn. 59: 103-113.

Rosenbaum, R.S., Ziegler, M., Winocur, G., Grady, C.L., and Moscovitch, M. 2004. "I have often walked down this street before": fMRI studies on the hippocampus and other structures during mental navigation of an old environment. Hippocampus 14: 826-835.

Scoville, W.B. and Milner, B. 1957. Loss of recent memory after bilateral hippocampal lesions. J. Neurol. Neurosurg. Psychiatr. 20: 11-21.

Shallice, T., Fletcher, P., Frith, C.D., Grasby, P., Frackowiak, R.S., and Dolan, R.J. 1994. Brain regions associated with acquisition and retrieval of verbal episodic memory. Nature 368: 633-635.

Shelton, A.L. and Gabrieli, J.D. 2002. Neural correlates of encoding space from route and survey perspectives. J. Neurosci. 22: 2711-2717.

Spiers, H.J., Burgess, N., Maguire, E.A., Baxendale, S.A., Hartley, T., Thompson, P.J., and O'Keefe, J. 2001. Unilateral temporal lobectomy patients show lateralized topographical and episodic memory deficits in a virtual town. Brain 124: 2476-2489.

Squire, L.R., Stark, C.E., and Clark, R.E. 2004. The medial temporal lobe. Annu. Rev. Neurosci. 27: 279-306.

Stark, C.E. and Squire, L.R. 2001. When zero is not zero: The problem of ambiguous baseline conditions in fMRI. Proc. Natl. Acad. Sci. 98: 12760-12766.

Stepankova, K., Fenton, A.A., Pastalkova, E., Kalina, M., and Bohbot, V.D. 2004. Object-location memory impairment in patients with thermal lesions to the right or left hippocampus. Neuropsychologia 42: 1017-1028.

Tulving, E. 1993. What is episodic memory? Current Perspec. Psychol. 2: 67-70.

Vargha-Khadem, F., Gadian, D.G., Watkins, K.E., Connelly, A., Van Paesschen, W., and Mishkin, M. 1997. Differential effects of early hippocampal pathology on episodic and semantic memory. Science 277: 376-380.

Willingham, D.B., Salidis, J., and Gabrieli, J.D. 2002. Direct comparison of neural systems mediating conscious and unconscious skill learning. J. Neurophysiol. 88: 1451-1460.

Wolbers, T. and Buchel, C. 2005. Dissociable retrosplenial and hippocampal contributions to successful formation of survey representations. J. Neurosci. 25: 3333-3340.

Woolrich, M.W., Ripley, B.D., Brady, M., and Smith, S.M. 2001 Temporal autocorrelation in univariate linear modeling of FMRI data. Neuroimage 14: 1370-1386.

Yonelinas, A.P., Kroll, N.E., Quamme, J.R., Lazzara, M.M., Sauve, M.J., Widaman, K.F., and Knight, R.T. 2002. Effects of extensive temporal lobe damage or mild hypoxia on recollection and familiarity. Nat. Neurosci. 5: 1236-1241.

Zola-Morgan, S., Squire, L.R., and Ramus, S.J. 1994. Severity of memory impairment in monkeys as a function of locus and extent of damage within the medial temporal lobe memory system. Hippocampus 4: 483-495.

Received March 12, 2007; accepted in revised form August 3, 2007. 


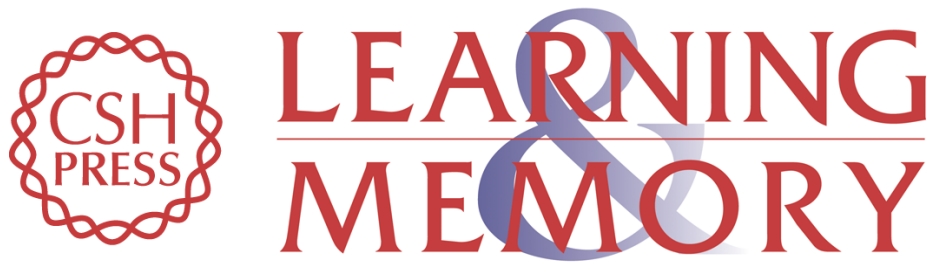

\section{Spatial and temporal episodic memory retrieval recruit dissociable functional networks in the human brain}

Arne D. Ekstrom and Susan Y. Bookheimer

Learn. Mem. 2007, 14:

Access the most recent version at doi:10.1101//m.575107

References This article cites 41 articles, 8 of which can be accessed free at:

http://learnmem.cshlp.org/content/14/10/645.full.html\#ref-list-1

License

Email Alerting Receive free email alerts when new articles cite this article - sign up in the box at the Service top right corner of the article or click here. 